\title{
Successful treatment of lung adenocarcinoma with gefitinib based on EGFR gene amplification
}

Chunguo Wang ${ }^{1}$, Feng $\mathrm{Xu}^{1}$, Jianfei Shen ${ }^{1}$, Linna Zhang ${ }^{2}$, Jian Zhang ${ }^{1}$, Jiang Jin ${ }^{1}$, Luca Ampollini ${ }^{3}$, Paul van Schil ${ }^{4}$, Hideharu Kimura ${ }^{5}$, Francesco Grossi ${ }^{6}$, Kenichi Suda ${ }^{7}$, Bo Zhang ${ }^{1}$, Dehua Ma ${ }^{1}$; written on behalf of the AME Lung Cancer Collaborative Group

${ }^{1}$ Department of Cardiothoracic Surgery, ${ }^{2}$ Department of Pathology, Taizhou Hospital of Zhejiang Province, Wenzhou Medical University, Linhai 317000, China; ${ }^{3}$ Thoracic Surgery, Department of Medicine and Surgery, University Hospital of Parma. Via Gramsci 14, 43126 Parma, Italy; ${ }^{4}$ Department of Thoracic and Vascular Surgery, Antwerp University Hospital, Edegem, Belgium; ${ }^{5}$ Department of Cellular Transplantation Biology, Kanazawa University Graduate School of Medicine, Kanazawa, Japan; ${ }^{6}$ Fondazione IRCCS Ca' Granda Ospedale Maggiore Policlinico, Milano, Italy; ${ }^{7}$ Division of Thoracic Surgery, Department of Surgery, Kinki University Faculty of Medicine, 377-2 Ohno-higashi, Osaka-Sayama, Japan Correspondence to: Bo Zhang; Dehua Ma. Department of Cardiothoracic Surgery, Taizhou Hospital of Zhejiang Province, Wenzhou Medical University, Linhai 317000, China. Email: zhbo1112@163.com; madh@enzemed.com.

Submitted Jul 20, 2018. Accepted for publication Oct 15, 2018.

doi: $10.21037 /$ jtd.2018.10.55

View this article at: http://dx.doi.org/10.21037/jtd.2018.10.55

\section{Introduction}

In the past, the optimal first-line treatment of advanced non-small cell lung cancer (NSCLC) patients with wildtype for epidermal growth factor receptor (wt-EGFR) has been platinum-based chemotherapy doublets $(1,2)$. More recently, clinicians have adopted immunotherapy with check-point inhibitors alone, in PD-L1 strong positive, or combined with chemotherapy independently to PD-L1 expression (3). In the second-line, for wt-EGFR patients, tyrosine kinase inhibitors (TKIs) have been considered, albeit controversially, as a potential treatment option $(4,5)$. Several studies have shown that EGFR gene copy number or amplification detected by fluorescence in situ hybridization (FISH) could be a good biomarker for predicting treatment response to EGFR TKIs in patients with advanced NSCLC (6-8). Here we reported a successful experience with a patient with lung adenocarcinoma wt-EGFR and EGFR gene amplification who received benefit from gefitinib treatment.

\section{Case presentation}

A 72-year-old, non-smoking man with paroxysmal cough and expectoration in the past 2 months was admitted to our hospital due to the detection from chest radiography of an abnormal nodular lesion on the left lung. Computed tomography (CT) showed a $2.3 \mathrm{~cm}$ nodule in the left upper lobe (Figure 1A). The patient underwent a videoassisted thoracoscopic left upper lobectomy and regional lymphadenectomy with a diagnosis of adenocarcinoma, pT2apN0M0 stage IB (Figure 1B). The patient was followed up regularly in an outpatient clinic without receiving adjuvant treatment. CT-scan performed during follow-up 5 years after the surgical resection revealed multiple nodules on the left lower lung, indicating a local recurrence of lung cancer (Figure 2A). Re-biopsy of one of the nodules was performed using CT-guided fine-needle aspiration, and the nodule was pathologically diagnosed as an intrapulmonary metastasis of the previous lung adenocarcinoma (Figure 2B). The mutation status of epidermal growth factor receptor (EGFR) exon 18 to 21 was assessed in the metastatic lesions but no mutation was found.

The patient received chemotherapy with cisplatin (1 cycle every 3 weeks, $25 \mathrm{mg} / \mathrm{m}^{2}$ on days 1 to 3 ) and gemcitabine $\left(1,000 \mathrm{mg} / \mathrm{m}^{2}\right.$ on days 1 and 8$)$ with a complete response assessed with CT after 4 cycles (Figure 3). Five months after first-line chemotherapy, the recurrent lesions were observed again on the left lower lobe but not on any other sites (Figure 3B). Subsequently, the patient was treated with 6 cycles of pemetrexed $500 \mathrm{mg} / \mathrm{m}^{2}$ on day 1 every 3 weeks. Unfortunately, the disease progressed on the left lower lobe (Figure 3C). Using a FISH test we found an EGFR gene 

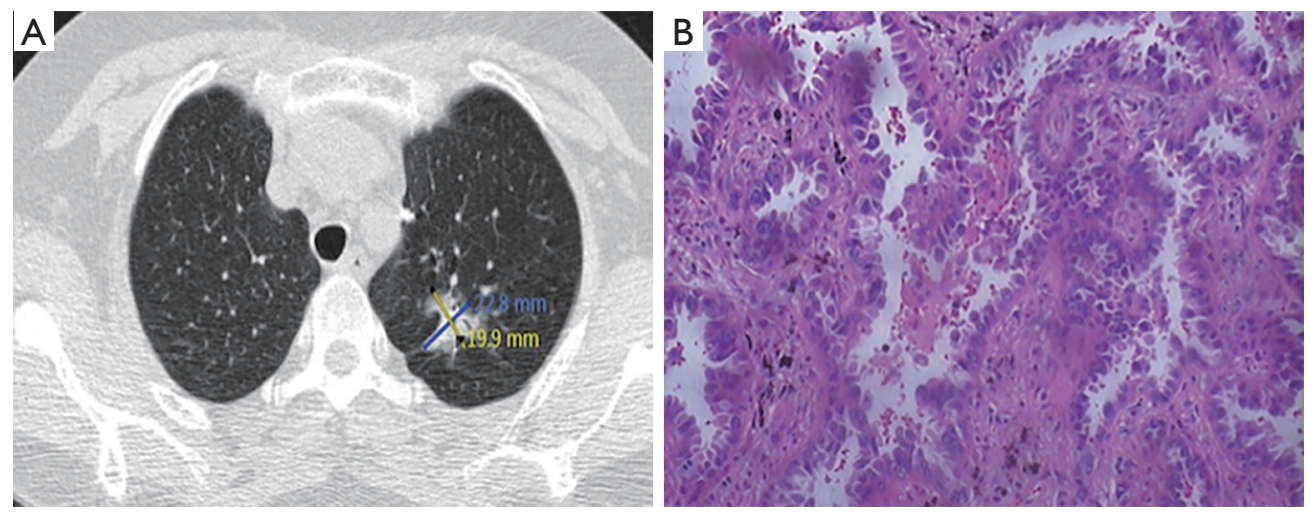

Figure 1 Chest CT findings and microscopic findings of the lung tumor. (A) CT shows a 2.3 cm nodule in the left upper lobe; (B) a hematoxylin and eosin stain $\times 100$ revealing an adenocarcinoma diagnosis. CT, computed tomography.
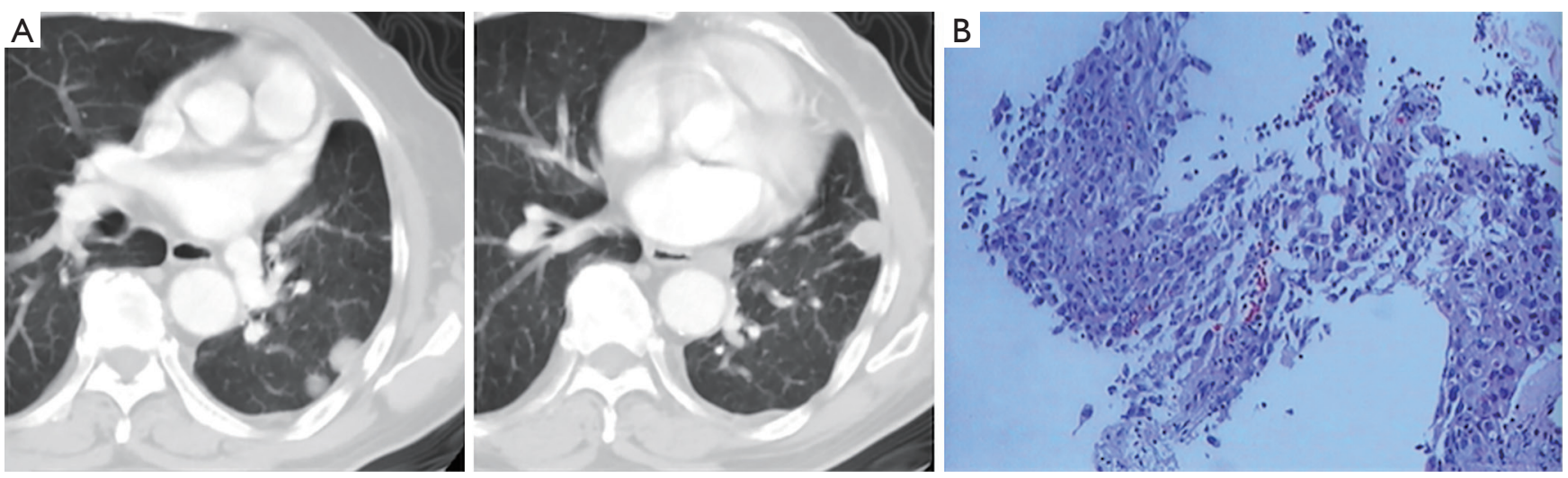

Figure 2 CT revealing small multiple nodules in the left lower lobe, with the largest diameter being about $1.2 \mathrm{~cm}$ and CT value of about $63 \mathrm{Hu}$ after enhancement (A). Pathologically diagnosed as an adenocarcinoma after re-biopsy $(\times 100)(\mathrm{B})$.

amplification in the tumor tissue in the last biopsy (Figure 4). Based on this result, as a third line treatment, the patient received a daily oral dose of $250 \mathrm{mg}$ of gefitinib. After 30 days of administration, CT revealed that the metastatic lesion on the left lower lobe had completely disappeared (Figure 3D), and metastatic lesions were not found in any other organs. The clinical evaluation indicated complete remission.

\section{Discussion}

Current literature and guidelines show that advanced NSCLC patients with activating EGFR mutations can derive significant benefit from EGFR-TKIs $(9,10)$. However, establishing a treatment for advanced NSCLC patients wild-type for EGFR still poses a significant challenge, and whether wt-EGFR patients should be treated with TKIs is still debatable. Two large, placebo-controlled phase III trials compared erlotinib or gefitinib $v s$. placebo in the second or third line setting in unselected patients with advanced NSCLC. The study showed that the subset of patients harboring high EGFR gene copy number may benefit from EGFR-TKI therapy $(11,12)$. Additionally, a phase II study investigated the activity and safety of afatinib in patients with advanced NSCLC with increased EGFR gene copy number and/or FISH with or without EGFR mutation (13). Cappuzzo et al. found that higher objective response rates (ORRs) were observed in the patients with gene amplification $(20.0 \%$; $=5$ of 25$)$ suggesting that EGFR FISH testing may identify an additional subset of patients with NSCLC who would benefit from first- or second-line afatinib therapy (13).

In the present case, re-biopsy of the left lung nodule suspected of being recurrent cancer was negative for EGFR 

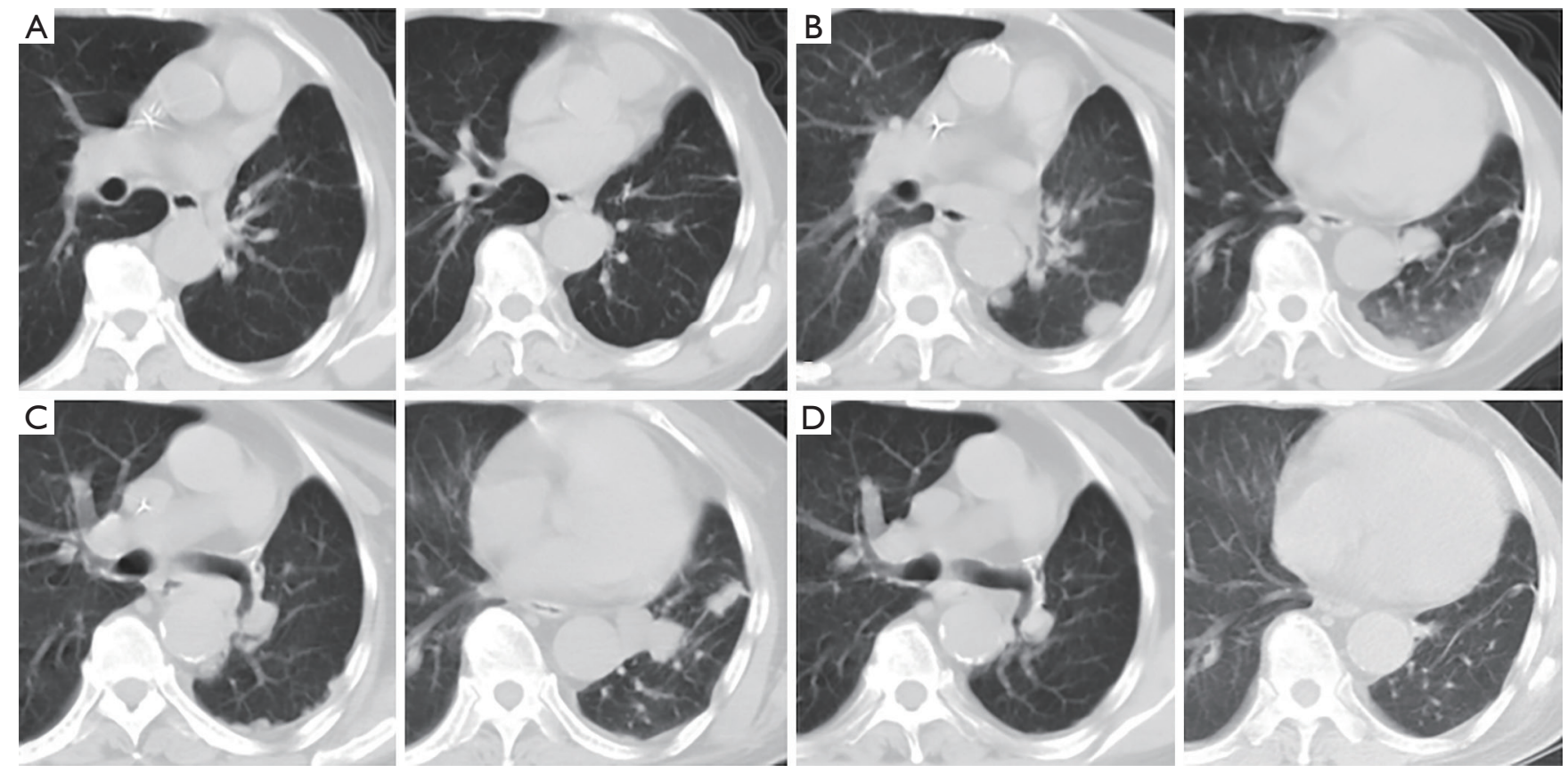

Figure 3 Imaging changes during treatment. (A) Absolute disappearance of all metastatic nodules after chemotherapy with cisplatin and gemcitabine; (B) recurrence of nodules in the left lower lobe after 5 months from the end of chemotherapy; (C) the primary metastatic lesions did not disappear, but new ones appeared after 6 cycles of pemetrexed monotherapy; (D) complete response of metastatic nodules after treatment with gefitinib.

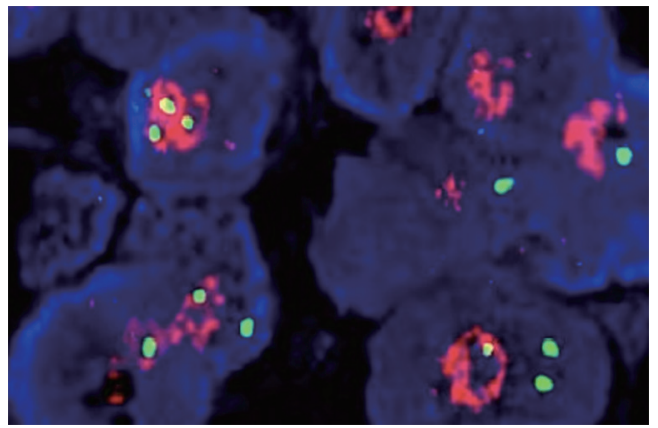

Figure 4 Amplification of EGFR in lung cancer revealed by fluorescence in situ hybridization (FISH) test. EGFR, epidermal growth factor receptor.

mutation. After the failure of chemotherapy, the EGFR gene amplification status was confirmed by FISH. Consistent with the current literature, the patient was administered gefitinib, and the clinical evaluation on CT was complete remission.

Several meta-analyses and clinical studies have also proposed that EGFR gene copy number detected by FISH is a candidate biomarker for predicting treatment response to EGFR-TKIs in patients with advanced NSCLC. Hirsch et al. (14) found that an increase in EGFR gene copy in the form of gene amplification correlates strongly with response, progression-free survival (PFS), and overall survival (OS) after treatment with gefitinib. Zhang et al. (15) conducted a meta-analysis, which included 17 studies with 2,047 patients, and they evaluated the relationship between EGFR gene copy number and EGFR-TKI treatment response in patients with advanced NSCLC. Their overall analysis revealed that EGFR gene copy number amplification was associated with higher ORR, OS, and PFS in patients with advanced NSCLC receiving TKIs. Wang et al. (16) reported that in patients with wild-type EGFR, EGFR FISH+ but not EGFR FISH- status correlated with longer PFS (4.4 vs. 2.0 months; $\mathrm{P}<0.001)$. This study suggests that EGFR gene copy number can be further detected in patients with wild-type EGFR, since patients in the FISH+ status can derive a greater benefit from EGFR-TKI.

Nevertheless, studies on EGFR gene copy number as a predictor of response to first-line therapy in advanced NSCLC with TKIs have shown inconsistent results. For instance, Fiala et al. (17) found no significant correlation 
between $E G F R$ gene amplification and survival, although they did observe a trend for longer PFS and OS in patients with EGFR amplification (3.9 and 13.6 months) compared to those without EGFR amplification (2.1 and 9.8 months).

\section{Conclusions}

In summary, the potential predictive value of gene copy number or amplification in response to TKIs therapy in advanced NSCLC with wt-EGFR remains up for debate. Despite this, EGRF-TKIs therapy still provides a possible therapeutic option for such cases.

\section{Acknowledgements}

None.

\section{Footnote}

Conflicts of Interest: The authors have no conflicts of interest to declare.

Informed Consent: Written informed consent was obtained from the patient for publication of this manuscript and any accompanying images.

\section{References}

1. Hamilton G, Rath B. Pharmacogenetics of platinum-based chemotherapy in non-small cell lung cancer: predictive validity of polymorphisms of ERCC1. Expert Opin Drug Metab Toxicol 2018;14:17-24.

2. Inno A, Di Noia V, D'Argento E, et al. State of the art of chemotherapy for the treatment of central nervous system metastases from non-small cell lung cancer. Transl Lung Cancer Res 2016;5:599-609.

3. Li J, Chen Y, Shi X, et al. A systematic and genomewide correlation meta-analysis of PD-L1 expression and targetable NSCLC driver genes. J Thorac Dis 2017;9:2560-71.

4. Lim SW, Park S, Kim Y, et al. Continuation of gefitinib beyond progression in patients with EGFR mutationpositive non-small-cell lung cancer: A phase II single-arm trial. Lung Cancer 2018;124:293-7.

5. Lisberg A, Garon EB. Epidermal growth factor tyrosine kinase inhibitor therapy inferior to second-line chemotherapy in EGFR wild-type non-small cell lung cancer patients: results of French nationwide observational study. Transl Lung Cancer Res 2017;6:S39-40.

6. McCoach CE, Le AT, Gowan K, et al. Resistance Mechanisms to Targeted Therapies in ROS1+ and ALK+ Non-small Cell Lung Cancer. Clin Cancer Res. 2018;24:3334-47.

7. Mitiushkina NV, Imyanitov EN. Distinct benefit from crizotinib in lung cancer patients carrying distinct ALK translocations: is fluorescent hybridization in situ testing still sufficient to guide clinical decisions? Transl Cancer Res 2016;5:S1393-5.

8. Barbareschi M, Barberis M, Buttitta F, et al. Predictive markers in lung cancer: a few hints for the practicing pathologist. Pathologica 2018;110:29-38.

9. Keedy VL, Temin S, Somerfield MR, et al. American Society of Clinical Oncology provisional clinical opinion: epidermal growth factor receptor (EGFR) Mutation testing for patients with advanced non-small-cell lung cancer considering first-line EGFR tyrosine kinase inhibitor therapy. J Clin Oncol 2011;29:2121-7.

10. Greenhalgh J, Dwan K, Boland A, et al. Firstline treatment of advanced epidermal growth factor receptor (EGFR) mutation positive non-squamous nonsmall cell lung cancer.Cochrane Database Syst Rev 2016;(5):CD010383.

11. Zhu CQ, Da Cunha Santos G, Ding K, et al. Role of KRAS and EGFR as biomarkers of response to erlotinib in National Cancer Institute of Canada clinical trials group study BR.21. J Clin Oncol 2008;26:4268-75.

12. Hirsch FR, Varella-Garcia M, Bunn PA, et al. Molecular predictors of outcome with gefitinib in a phase III placebocontrolled study in advanced non-small-cell lung cancer. J Clin Oncol 2006;24:5034-42.

13. Cappuzzo F, Finocchiaro G, Grossi F, et al. Phase II study of afatinib, an irreversible ErbB family blocker, in EGFR FISH-positive non-small-cell lung cancer. J Thorac Oncol 2015;10:665-72.

14. Hirsch FR, Varella-Garcia M, McCoy J, et al. Increased epidermal growth factor receptor gene copy number detected by fluorescence in situ hybridization associates with increased sensitivity to gefitinib in patients with bronchioloalveolar carcinoma subtypes: a Southwest Oncology Group Study. J Clin Oncol 2005;23:6838-45.

15. Zhang X, Zhang Y, Tang H, et al. EGFR gene copy number as a predictive/biomarker for patients with nonsmall-cell lung cancer receiving tyrosine kinase inhibitor treatment: a systematic review and meta-analysis. J Investig Med 2017;65:72-81.

16. Wang F, Fu S, Shao Q, et al. High EGFR copy number 
predicts benefits from tyrosine kinase inhibitor treatment for non-small cell lung cancer patients. with wild-type EGFR. J Transl Med 2013;11:90.

Cite this article as: Wang C, Xu F, Shen J, Zhang L, Zhang J, Jin J, Ampollini L, van Schil P, Kimura H, Grossi F, Suda K, Zhang B, Ma D; written on behalf of the AME Lung Cancer Collaborative Group. Successful treatment of lung adenocarcinoma with gefitinib based on EGFR gene amplification. J Thorac Dis 2018;10(11):E779-E783. doi: $10.21037 /$ jtd.2018.10.55
17. Fiala O, Pesek M, Finek J, et al. Epidermal Growth Factor Receptor Gene Amplification in Patients with Advancedstage NSCLC. Anticancer Res 2016;36:455-60. 